https://idp.uoc.edu

ARTÍCULO

\title{
Análisis del desarrollo normativo de las criptomonedas en las principales jurisdicciones: Europa, Estados Unidos y Japón
}

\author{
Miguel Ángel García-Ramos Lucero \\ EAE Business School
}

\author{
Ricardo Rejas Muslera \\ Universidad Camilo José Cela
}

Fecha de presentación: agosto de 2021 Fecha de aceptación: octubre de 2021 Fecha de publicación: marzo de 2022

\section{Resumen}

Las criptomonedas o divisas digitales son un nuevo tipo de activo digital que se puede utilizar en múltiples aplicaciones, como transferir dinero e invertir. A diferencia de la mayoría de las empresas en las que invertir, las criptomonedas carecen de una entidad legal específica a cargo de la protección del consumidor. El carácter virtual y descentralizado de esta tecnología imposibilita la aplicación de los marcos legales tradicionales. Además, la ausencia de una entidad legal específica hace que la aplicación de cualquier nuevo marco legal sea poco confiable. Por estas dos razones, con independencia de su naturaleza, el estado regulatorio actual de las criptomonedas genera muchas dudas. Este artículo pretende una descripción analítica de los diferentes enfoques que han adoptado las entidades legislativas en las principales economías (Unión Europea, Estados Unidos y Japón) para hacer frente a los desafíos que suponen las criptomonedas. El artículo pretende mostrar los pros y los contras de cada enfoque y mostrar las corrientes de opinión reciente del mundo académico-científico.

\section{Palabras clave}

criptomoneda; mercado financiero; legislación; regulación; leyes 


\title{
Analysis of the normative development of cryptocurrencies in the main jurisdictions: Europe, US and Japan
}

\begin{abstract}
Cryptocurrencies or digital currencies are a new type of digital asset that can be used in many applications such as transferring money and investing. Unlike most companies you can invest in, cryptocurrencies do not have a specific legal entity in charge of consumer protection. The virtual and decentralized nature of this technology makes it impossible to apply traditional legal frameworks. In addition, the absence of a specific legal entity makes the application of any new legal framework unreliable. For these two reasons, the current regulatory status of cryptocurrencies or digital currencies generates doubt and hesitation. This article seeks to describe analytically the different approaches adopted by legislative entities in the main economies (European Union, United States and Japan) to face the challenges posed by cryptocurrencies. The article aims to show the pros and cons of each approach and exhibit recent discussions in the academic-scientific world.
\end{abstract}

\section{Keywords}

cryptocurrency; financial market; crypto-legislation; regulation; laws 


\section{Introducción}

La criptomoneda es una innovación de vanguardia en tecnología financiera dentro del denominado «Fintech» (finanzas y tecnología) y actualmente es un foco de atención en todo el mundo por las oportunidades y amenazas que genera (Noya, 2016). Supone una revolución que cambiará el escenario financiero, económico, político y social. Sin embargo, su rápido desarrollo ya ha provocado una serie de eventos o escándalos de seguridad pública en todo el mundo (Liang et al., 2019). La criptomoneda se creó originalmente como una posible implementación de una moneda o medio de pago digital descentralizado, lo cual significa que no se rige por ninguna administración pública.

Desde el punto de vista fiscal y del gravamen sobre estas u operaciones realizadas con este aspecto colateral, los enfoques hacia las monedas virtuales difieren entre jurisdicciones; al igual que las definiciones de monedas virtuales de un país a otro (Bhatia, 2021). Esta definición condiciona si la regulación tributaria puede abarcar las criptomonedas. La mayoría de las veces, las monedas virtuales se incluyen en una determinada categoría de ingresos y, por tanto, están sujetas a un impuesto correspondiente (Kazakov, 2018).

Numerosos Estados también han publicado documentos aclaratorios sobre el tratamiento fiscal de las monedas virtuales y cómo se les aplica el marco legal nacional existente. Solamente un Estado considera las criptomonedas como una moneda adicional de curso legal además de la regular fiat oficial (Ana et al., 2018). En este sentido, la materia analizada se encuentra de actualidad ya que EI Salvador, desde el pasado 7 de septiembre de 2021, es la primera nación que ha adoptado el bitcóin como moneda de curso legal.

En este aspecto, se puede resaltar una falta general de coherencia en el enfoque con el que se administran y regulan las monedas virtuales. Se intentó vincularlas con las monedas convencionales, pero las características tecnológicas que aportan un mayor anonimato y falta de intermediarios hicieron de la regulación y estandarización del manejo de este instrumento financiero un verdadero reto para las autoridades en casi todos los países del mundo (Bolotaeva et al., 2019). Solo unos pocos países colocan las monedas virtuales bajo el mismo régimen fiscal que las monedas regulares (Vargas, 2020).
En numerosas otras jurisdicciones nacionales, existe una elevada incertidumbre sobre la definición de monedas virtuales, lo que lleva a diferentes interpretaciones de su régimen fiscal (Sanz-Bas et al., 2021) por la falta de madurez de su marco regulatorio. Las criptomonedas generalmente se consideran una forma de propiedad intangible o activo financiero en lugar de una moneda. Por ello, su gravamen suele ser vía impuesto sobre la propiedad y no mediante el impuesto sobre la renta. La regulación cambiaria a menudo contiene disposiciones que minimizan las consecuencias fiscales de los impuestos para las personas físicas o los pequeños comerciantes, por ejemplo, al limitar las transacciones en cuentas individuales (Jiménez, s. f.).

Para ello, el presente artículo se iniciará profundizando en el origen de las criptomonedas, para posteriormente conceptualizar su definición y estudiarlas como una herramienta económica de inversión alternativa. Finalizaremos con el reto que supone para los Gobiernos el uso de estas.

La metodología utilizada para la recopilación de información y el posterior desarrollo descriptivo de los enfoques regulatorios ha sido el siguiente. El diseño del estudio consistió en desarrollar una revisión bibliográfica durante los meses de enero a junio de 2021 de publicaciones registradas en la base de datos de Scopus, Google Académico y ResearchGate.

El paradigma de este artículo científico es de modo descriptivo e interpretativo, puesto que su interés ha sido comprender e interpretar la metodología constructiva y holística de la legislación ante una revolución tecnológica sin precedentes que puede cambiar todos los pilares económicos y sociales conocidos hasta el momento. La tecnología supone un enorme desafío, principalmente su utilización minorista, y la irrupción de las divisas digitales plantea la necesidad de resolver cuestiones tributarias, derecho de propiedad, contratos, quiebras, liquidaciones y protección de datos.

La causalidad de esta es prospectiva y su axiología tiene en cuenta los valores, puesto que estos influyen en la solución del problema, la teoría, el método y el análisis.

Respecto a la metodología, los términos utilizados para la búsqueda fueron «criptomoneda» (AND) «criptolegislación», «mercado financiero», «regulación» y «leyes». Se utilizaron diversas combinaciones de estos descriptores 
para realizar una búsqueda más exhaustiva y completa. A dichas combinaciones se añadió el país en el que se quería centrar la búsqueda (España, Estados Unidos, Japón):

- Criptomoneda AND criptolegislación

- Criptomoneda AND mercado financiero

- Criptomoneda AND regulación

- Criptomoneda AND leyes

De cara a seleccionar las publicaciones encontradas, la técnica de filtrado de información se llevó a cabo mediante un filtro basado en criterios de inclusión y exclusión. Los criterios de inclusión fueron:

- Estudios publicados desde 2010 en adelante.

- Artículos publicados en inglés o en español.

Mientras que los criterios de exclusión fueron los siguientes:

- Artículos a los que no se tuvo acceso a texto completo.

- Artículos que no se centrasen en el tema.

- Artículos repetidos.

En total, se obtuvieron 98 artículos como resultados de la búsqueda realizada, de los cuales, tras aplicar los criterios de inclusión y exclusión previamente señalados, solo 20 se consideraron válidos para dar respuesta al objetivo del presente trabajo.

\section{Criptomonedas}

Después de veinte años de intentos fallidos de crear una moneda virtual privada, el bitcóin emergió tras la crisis financiera global de 2008 (el 31 de octubre de 2008, dos meses después de la suspensión de pagos de Lehman Brothers, el usuario Nakamoto publicó el artículo que dio nacimiento al bitcóin). El/los creador/es del bitcóin, del/de los que aún se desconoce su verdadera identidad, estaba/n decidido/s a proporcionar medios descentralizados, privados y seguros para transferir valor a través de internet sin la intervención de instituciones soberanas, bancos centrales o intermediarios financieros (Zaera Vidal, 2014). Las monedas virtuales, también denominadas criptomonedas, pueden definirse (según GAFI, Grupo de Acción Financiera
Internacional) «como una representación digital de valor no emitida ni garantizada por un banco central ni por una autoridad pública, no necesariamente asociada a una moneda de curso legal, que no tiene la consideración de moneda o divisa, pero es aceptada por personas físicas o jurídicas como medio de pago y que puede transferirse, almacenarse o negociarse por medios electrónicos».

Las criptomonedas y su tecnología subyacente blockchain se utilizan en varias aplicaciones diferentes, que incluyen pago de impuestos, dinero virtual, autenticación de identidad y juegos de azar, entre otras. Estas aplicaciones dependen de la capacidad del sistema para registrar, transferir y almacenar datos de forma segura en libros de contabilidad distribuidos Ilamados blockchain (Zaera Vidal, 2014). El bitcóin es un ejemplo de criptomoneda. Sin embargo, existen muchas variedades de criptomonedas (en la actualidad hay más de 8.500, aunque 20 de ellas representan el $90 \%$ de su valor de mercado, cuya capitalización creció un 300 \% en 2020), como Ethereum, Ripple, Litecoin, Dash y Metal.

Cada criptomoneda tiene características únicas que plantean desafíos únicos a los reguladores. Una de las principales dificultades legales asociadas con la regulación de las criptomonedas es que son de naturaleza incorpórea. El aspecto intangible de las monedas virtuales crea problemas de debido proceso y cortesía (Sereda, 2020).

Si se supone que las criptomonedas se almacenan en servidores físicos en todo el mundo, y si los tribunales pueden determinar bitcoines en una ubicación física específica, entonces se aplican las reglas de derecho civil existentes (Novikov, 2018). Sin embargo, la estructura de datos distribuidos de la tecnología blockchain hace que sea imposible determinar la ubicación específica de cualquier dato en particular.

Incluso digitalmente, los bitcoines no existen como monedas fungibles con atributos y números de seguimiento únicos. En cambio, cada bitcóin es una secuencia de firmas realizadas por claves privadas criptográficas controladas por usuarios de bitcoines. La naturaleza revolucionaria de la tecnología es la razón principal por la que no es fácil encajar en el marco legal existente (Lishchynska, 2019). 


\section{Regulación de las criptomonedas}

Con objeto de abordar la naturaleza jurídica de las criptomonedas, son posibles dos enfoques. Por un lado, equipararlas a los objetos de regulación legal ya existentes (títulos de anotaciones en cuenta, moneda tradicional, bienes, etc.; podemos definirlo como un enfoque statu quo), lo que implica la necesidad de crear y adaptar solo ciertas regulaciones que han de tener en cuenta las características específicas de la criptomoneda como variante del objeto correspondiente. Por otro lado, se puede introducir desde cero el concepto de criptomoneda en la legislación actual, reconociéndola como un objeto fundamentalmente nuevo de regulación legal y, como resultado, crear una legislación sobre criptomonedas desde el inicio (Samolysov, 2020).

Hoy en día, la mayoría de los países del mundo han abordado la disrupción de las divisas digitales y están tratando de regular las relaciones de criptomonedas, enfocándose en la mayoría de las ocasiones en la concesión de licencias de transacciones de criptomonedas, su gravamen (Bal, 2014), así como en contrarrestar su carácter anónimo derivado de la criptografía y su utilización por el cibercrimen y la financiación del terrorismo (Bălășoiu, 2021).

Al mismo tiempo, las agencias gubernamentales a menudo no tienen una posición clara ni uniforme con respecto a la naturaleza legal de las criptomonedas y, por tanto, intentan regular su circulación con inseguridad con relación a esta revolución tecnológica (Liang et al., 2019). En muchas ocasiones, el derecho tradicional carece de una respuesta coherente y transversal a este desafío tecnológico. Cabe destacar que los intentos de regular de alguna manera las relaciones legales asociadas con la criptomoneda no tienen sentido hasta que los derechos digitales, el dinero y los contratos se conviertan en objetos de derechos civiles.

Una de las opciones para determinar la naturaleza jurídica de la criptomoneda, que ha sido propuesta reiteradamente por funcionarios de diferentes países, es la introducción del concepto de bienes digitales o bienes virtuales. Cuando se adopte esta opción, la legislación civil debería complementarse con normas sobre un nuevo objeto de los derechos civiles: las cosas digitales (virtuales) (Bolotaeva et al., 2019). Sin embargo, es difícil extender el régimen de derechos de propiedad a tal objeto, ya que es intangible.
Desde el punto de vista del derecho civil, solo una cosa material está sujeta a enajenación, mientras que los objetos intangibles de los derechos civiles, incluidos los resultados de la actividad intelectual y los medios de individualización de personas jurídicas, bienes, obras, servicios y empresas, no lo están. Es decir, solo los derechos sobre ellos están sujetos a enajenación. Varios países han llegado a comprender gradualmente que es incorrecto identificar la criptomoneda con objetos de circulación civil ya existentes (Skorokhod et al., 2016).

Los puntos de vista discutidos anteriormente reflejan las diversas propiedades y funciones de la criptomoneda. Por un lado, la criptomoneda puede verse como una mercancía, ya que tiene un valor real, que consiste en los costes de su producción en forma de uso de la potencia de cómputo de las computadoras y, en consecuencia, el de la electricidad, que es significativo. Por otro lado, la clasificación de la criptomoneda como valores no certificados también es posible, ya que, al fijar un valor determinado, certifica el derecho del propietario a recibir una determinada cantidad de dinero (derecho de reclamación) (Sanz-Bas et al., 2021). Sin embargo, la criptomoneda también tiene características que no permiten categorizarla de manera inequívoca.

Asimismo, si el usuario es una de las partes de la relación jurídica, en los sistemas de dinero electrónico, este es un operador del sistema, y en un sistema de criptomonedas descentralizado esto no es por definición. Si no hay deudor, la representación digital del valor existe desde antes de la llegada de las criptomonedas. Así es como funciona el dinero electrónico, que se utiliza como sistemas tradicionales de almacenamiento de moneda. Sin embargo, a diferencia de la criptomoneda, que no está garantizada por nada y no está protegida por ninguna propiedad, el dinero electrónico está protegido por dinero fiduciario real (Moorthy, 2018).

La capacidad de pago de una criptomoneda se sustenta únicamente en su reconocimiento por parte de los participantes en la facturación y el mecanismo económico de oferta y demanda, y la liberación de nuevas unidades monetarias, a diferencia de las emisiones tradicionales, se realiza conectando la potencia de cómputo adicional al sistema a través de dichos participantes (Jiménez, s. f.). Esta propiedad, por sí sola, es una base suficiente para comprender que entre los objetos de regulación legal existentes es imposible encontrar análogos de la criptomoneda. 
La criptomoneda es distintiva y única, y sus propiedades inherentes deben ser la base de su estatus y regulación legales de las relaciones con su uso. Los países que han aceptado el surgimiento de la criptomoneda como una realidad y se han dado cuenta de la irracionalidad de su prohibición legislativa también deben reconocer que el nuevo objeto de regulación legal afectará, en cierta medida, a todas las relaciones sociales existentes (Novikov, 2018).

Por tanto, se necesita un enfoque equilibrado para determinar la naturaleza jurídica y el estado legal de la criptomoneda. Esto determinará el contenido de las normas legales sobre el control de su facturación, impuestos, licencias de las actividades de los participantes en las relaciones, etc. Cabe tener en cuenta que la legitimación de las funciones de la criptomoneda también incrementará la eficiencia de la inteligencia financiera, ya que será posible adaptar estándares y recomendaciones modernas para combatir el lavado de dinero, teniendo en cuenta las peculiaridades de la moneda virtual (Samolysov, 2020).

Por tanto, como conclusión, las diferentes regulaciones nacionales han adoptado distintos enfoques para afrontar la cobertura legal de este fenómeno de la tecnología. Esto es consecuencia de la dificultad de la determinación de la naturaleza. Las diferentes estrategias pueden resumirse en:

- La prohibición, tanto de las emisiones (ICO) como de las operaciones con activos digitales. La ventaja es otorgar certidumbre a un bajo coste regulatorio, mientras que su desventaja es que desincentiva el desarrollo e incentiva el uso clandestino. China es el principal ejemplo de regulación que adoptó esta postura.

- La suposición de que estos activos digitales representan un activo distinto a los tradicionales, con difícil encaje, por lo que debe regularse específicamente en cada jurisdicción hasta que haya una regulación internacional madura uniforme. Ejemplos de este modelo son Francia, Israel, Malta, Méjico o Tailandia.

- La adaptación de la normativa existente con anterioridad en materia de valores o instrumentos financieros hasta la maduración de una legislación internacional ampliamente aceptada. Aunque no es un nuevo cuerpo regulatorio, en ocasiones se realizan ajustes de las normas. Son ejemplos de esto España, Alemania o el Reino Unido.
Por tanto, el uso de las criptomonedas presenta un elevado nivel de desafío a las autoridades en cuanto al equilibrio entre la estabilidad financiera y el incentivo al desarrollo tecnológico. Las divisas digitales, sin duda, plantean nuevos retos y pueden obligar a las administraciones reguladoras a la adopción de nuevas metodologías y desarrollos regulatorios. A modo de ejercicio de síntesis, los principales retos pueden catalogarse como:

- Regulación AML y KYC (anti money laundering y know your customer). La naturaleza anónima de esta nueva categoría de activos dificulta la determinación sobre su grado de uso en la evasión fiscal o el intercambio en actividades ilícitas.

- Vacío legal en la regulación de los mercados secundarios privados de criptomonedas y monederos que han derivado ocasionalmente en estafas a los usuarios finales. Resulta en cierta medida paradójico, ya que los activos digitales tuvieron su cuna en la desconfianza de los usuarios en el sistema financiero tradicional en la crisis financiera de 2008. Actividades fraudulentas han acontecido igualmente en los mercados primarios (ICO), en ocasiones mediante emisiones realizadas por start-ups con estados financieros falseados y actividades empresariales opacas.

- Posible riesgo sistémico, vulnerabilidad del sistema financiero global. Esto supone un salto cualitativo en el nivel de riesgo y obliga a las autoridades a elevar el grado de supervisión y la digitalización e inversión en software por parte de la Administración pública.

\section{Resultados}

\subsection{Legislación europea}

El uso de criptomonedas no siempre es legal y, a pesar de la seguridad obvia que brindan las monedas virtuales, encontramos que se usan de manera fraudulenta. Por ello, la legislación ha ido desarrollándose y adaptándose de manera gradual (Sanz-Bas et al., 2021).

Con respecto a la Unión Europea, la Directiva (UE) 2018/843 (UE 2018), también conocida como la «Quinta Directiva», fue adoptada el 30 de mayo de 2018 para pre- 
venir el uso del sistema financiero con fines de blanqueo de capitales, estableciendo conceptos relacionados con las monedas virtuales y orientando a los países sobre cuáles deberían ser las reglas nacionales (Skorokhod et al., 2016). Esta directiva de la UE fija la fecha para su transferencia en los Estados miembros el 10 de enero de 2020.

Además, el GAFI (Grupo de Acción Financiera Internacional) publicó directrices sobre activos virtuales y proveedores de servicios en 2019. Esta organización a menudo hace recomendaciones, publicaciones, informes y evaluaciones para ciertos países, que conducen a normas europeas, que, a su vez, se materializan en la legislación nacional. Así, la información sobre las publicaciones e informes del GAFI nos permite saber qué camino tomará el sistema financiero español. En este sentido, las siguientes serán publicaciones de referencia a nivel internacional, europeo y nacional, respectivamente, sobre la regulación de la moneda virtual (Skorokhod et al., 2016):

- Directrices del GAFI sobre activos virtuales y proveedores de servicios, publicadas en junio de 2019.

- Quinta Directiva 2018/843 UE sobre prevención del blanqueo de capitales.

- Real Decreto Ley 7/2021 de transferencia de directivas de la UE, incluida la prevención del blanqueo de capitales a partir de 2021.

- Borrador del reglamento europeo relativo a los mercados de criptoactivos (MiCA).

La Guía de Activos Virtuales y Proveedores de Servicios de GAFI (2019) nos da dos definiciones importantes como activo virtual (VA) y proveedor de servicios de activos virtuales (VASP) (Sanz-Bas et al., 2021). La primera es una representación digital de valor que puede venderse o transferirse digitalmente y utilizarse para pagos o con fines de inversión. Además, VASP es una persona física o jurídica que no está cubierta por otras pautas y realiza cualquiera de las siguientes acciones: intercambio entre activos virtuales y monedas fiduciarias; intercambio entre una o más formas de activos virtuales, y transferencia de activos virtuales, así como la prestación de servicios financieros relacionados con la oferta o la venta de un activo virtual.

Asimismo, la Quinta Directiva 2018/843 del Parlamento Europeo y del Consejo, del 30 de mayo de 2018 (UE 2018), sobre la prevención del uso del sistema financiero con fi- nes de blanqueo de capitales o financiación del terrorismo, y que modifica las directivas 2009/138 y EC 2013/36UE, reflejan varios aspectos de las criptomonedas que se han trasladado a la actual legislación (Skorokhod et al., 2016):

- Incluye nuevas partes obligadas: moneda virtual a proveedores de servicios de cambio fiduciario. Monedas (operadores de intercambio de criptomonedas) y proveedores de servicios para almacenar billeteras electrónicas (organizaciones que brindan servicios para proteger claves criptográficas privadas en nombre de sus clientes, para almacenar y transferir monedas virtuales).

- Tiene como objetivo conseguir que los proveedores denuncien transacciones sospechosas, además de limitar parcialmente el anonimato que permiten las criptomonedas. Las autoridades competentes deben estar facultadas, mediante personas autorizadas, para monitorear el uso de monedas virtuales.

- Otra medida con relación a estas nuevas entidades es la obligación de registrarlas, aunque no se especifica en qué forma de registro ni en sus condiciones. La propia directiva fija la fecha del 10 de enero de 2020 para su transposición.

\subsection{Legislación de Estados Unidos}

La naturaleza seudónima de la criptomoneda permite que las transacciones transfronterizas eludan las reglas de Know-Your-Customer (KYC) (conocer a tu cliente) y las medidas contra el Anti-Money Laundering (AML) (blanqueo de dinero) (Hughes, 2017). La capacidad de rastrear cada transacción sin poder determinar su remitente cambiará las interrelaciones ciudadano-Estado. La capacidad de encubrir la riqueza al Gobierno puede representar un gran desafío para los modelos de regímenes políticos existentes y su capacidad fiscal recaudatoria.

En primer lugar, la Comisión de Comercio de Commodities (CFTC) participa activamente en la aplicación de las regulaciones en las operaciones de compraventa de bitcoines. Varios exchanges (mercados secundarios) ofrecen trading (operaciones de compra o venta) y brokerage apalancado y varios intermediarios o brókeres ofrecen contratos de futuros de bitcoines. Sin embargo, los inversores minoristas estadounidenses que utilizan estos sitios web pueden 
infringir las reglas de la CFTC (Moorthy, 2018). La CFTC establece que los inversores minoristas estadounidenses pueden comprar derivados apalancados en un mercado regulado, pero no en el extranjero.

Además, la CTFC anunció que el bitcóin y las monedas virtuales no son monedas extranjeras. En consecuencia, estas tecnologías no cumplen plenamente con las reglas de la CFTC para la negociación de derivados financieros (Hughes, 2017). En lugar de considerarlas una moneda extranjera, la CFTC declaró en septiembre de 2015 que el bitcóin y las monedas virtuales son un tipo de mercancía. En el artículo 1a (9) de la Ley de intercambios de productos básicos (CEA), un producto básico o commodity se define como todos los servicios, derechos e intereses con respecto a los cuales se celebran contratos para un suministro futuro, ahora o en el futuro.

En segundo lugar, a nivel federal, la Red de Ejecución de Delitos Financieros (FinCEN) es muy activa en la regulación de las monedas virtuales. FinCEN ha publicado varios fallos y dictámenes administrativos en casos de moneda virtual en Internet. En general, cualquier empresa que transfiera fondos de una persona a otra generalmente requiere una licencia de transferencia de dinero. Dado que las redes de criptomonedas permiten a los usuarios transferir fondos de una persona a otra, las empresas que facilitan estas transacciones requieren una licencia de transferencia de dinero (Sereda, 2020).

Algunos de los primeros casos judiciales involucraron a empresas o personas que no tenían una licencia de transferencia de dinero antes de facilitar las transacciones con criptomonedas. El 18 de marzo de 2013, FinCEN anunció que la Ley de Secreto Bancario (BSA) se aplica a los consumidores y empresas involucradas en el ecosistema de criptomonedas (Sereda, 2020). Esto significa que los diferentes actores de la industria de criptomonedas deben registrarse con FinCEN como una empresa de servicios monetarios (MSB, money services business) y que cualquier empresa que opere en el network o red de las divisas digitales debe cumplir con las normas AML y KYC.

De acuerdo con las reglas MSB de FinCEN, los diferentes operadores de los mercados secundarios de criptomonedas deben permitir que el Gobierno federal acceda de manera transparente a los registros comerciales. Además, la empresa de criptomonedas es responsable de informar periódicamente a FinCEN, y esta puede realizar auditorías selectivas de la empresa. Esta guía, proporcionada por FinCEN, establece explícitamente que los consumidores de criptomonedas no se consideran MSB y, por tanto, las reglas no se aplican específicamente a las personas (Bălășoiu, 2021).

En enero de 2014, FinCEN publicó una guía para aclaraciones adicionales a las pautas de moneda virtual de 2013. Según FinCEN, los mineros o verificadores de transacciones en una red de consenso de prueba de trabajo ( $P$ oWProof of Work) descentralizada (algoritmo de consenso original en blockchain) en la mayoría de los casos no están obligados a registrarse como MSB (Hughes, 2017). La minería de criptomonedas es el conjunto de ejercicios necesarios para la validación y el procesamiento de las transacciones del activo digital. En el caso del bitcóin, en la cadena de bloques, por la cual los mineros obtienen bitcoines como recompensa. Además, las empresas que desarrollan software que permite a los usuarios comerciar con criptomonedas tampoco están obligadas a registrarse como MSB en FinCEN.

Finalmente, en 2016, FinCEN publicó una guía adicional sobre cómo se pueden utilizar las criptomonedas para promover el terrorismo y el ciberdelito. En mayo de 2016, la directora de FinCEN, Jennifer Shaski Culvery, declaró que la regulación de la moneda virtual de FinCEN está tratando de equilibrar los intereses de prevenir el terrorismo y el apoyo a la innovación en tecnología financiera (Hughes, 2017). En esa guía también se explica cómo se pueden utilizar las criptomonedas para promover el terrorismo y el ciberdelito. FinCEN dijo que para evitar que las monedas virtuales se conviertan en una herramienta para el crimen, las empresas y las agencias gubernamentales deben compartir información sobre actividades sospechosas asociadas con este nuevo sistema de pago.

En tercer lugar, según el Servicio de Impuestos Internos de Estados Unidos (IRS, Internal Revenue Service), las plusvalías de las inversiones en moneda virtual están gravadas sobre las ganancias de capital (Braaten y Vaughn, 2019).

El 25 de marzo de 2014, el IRS emitió el documento de orientación IR-2014-36, que establece que una persona que «extrae» o mina moneda virtual como comercio o negocio se encontrará sujeta al impuesto sobre el trabajo por cuenta propia (Konowicz, 2018). Sin embargo, el Go- 
bierno declara que la moneda virtual no está clasificada como una moneda que pueda generar ganancias o pérdidas cambiarias para propósitos de impuestos federales de Estados Unidos.

En este documento, el IRS establece que las criptomonedas se clasificarán como propiedad a efectos fiscales federales. Esta guía establece que los principios fiscales generales aplicables a las transacciones inmobiliarias regulares también se aplican a las transacciones de moneda virtual (Braaten y Vaughn, 2019).

En consecuencia, las empresas que aceptan bitcoines y otras criptomonedas para pagar bienes y servicios deben pagar impuestos sobre la renta en relación con los pagos. Cuando se utilizan criptomonedas para pagos, se aplican las políticas del IRS con respecto a la provisión de información sobre transacciones inmobiliarias (Konowicz, 2018). Las empresas que pagan salarios a los empleados en bitcóin están sujetas a impuestos sobre los empleados y el empleador debe incluirlas en un formulario W-2 (certificado de retenciones en Estados Unidos para las declaraciones federales y estatales).

Además, los impuestos sobre la nómina y los impuestos sobre la renta federales se aplican a los salarios pagados en criptomonedas. Los empleadores que contratan a autónomos o contratistas independientes, así como los trabajadores autónomos, deben completar el Formulario 1.099 para declarar sus ingresos en criptomonedas (Hughes, 2017).

Para la determinación de la base imponible por los ingresos de bitcoines, el IRS establece que los contribuyentes deben cuantificar el valor justo de mercado de los bitcoines en el momento del pago (Braaten y Vaughn, 2019). Además de la orientación proporcionada por el IRS, el Departamento de Justicia también ayuda al IRS a monitorizar a los usuarios que no cumplen con las obligaciones fiscales por las plusvalías generadas. Los detalles de los procedimientos del DOJ (Department of Justice) con respecto a esta posible evasión de impuestos se establecen en la sección DOJ del Capítulo II, Parte C.

\subsection{Legislación en Japón}

En Japón, la criptomoneda se considera un activo denominado en moneda, pero no puede considerarse moneda de curso legal. Por ello, se desarrolló un conjunto de reglas para la moneda digital con la promulgación de la Ley de Moneda Virtual en abril de 2017 mediante la aprobación de la Agencia de Servicios Financieros (JFSA, Financial Services Agency) (Moorthy, 2018).

Según esta ley, el uso de bitcóin y Ethereum se reconoce como un método de pago legal. Independientemente, el hecho de que ambas criptomonedas estén exentas del impuesto al consumo japonés muestra que estas monedas digitales no obtienen el mismo estatus que las monedas fiduciarias existentes, como el yen (Cvetkova, 2018).

Sin embargo, se han realizado otras modificaciones a esta legislación. El requisito de registrar los cambios de moneda virtual con la JFSA es uno de ellos (Arora, 2020). Esto impone al intercambiador de divisas virtual la obligación de llevar a cabo la diligencia debida de KYC (know your customer) sobre sus clientes.

Además de lo anterior, existen algunos otros requisitos estrictos que debe cumplir cada moneda virtual, como tener un capital líquido mínimo de 10 millones de yenes, implementar un programa seguro de administración de TI (tecnología de la información) para protegerse contra la piratería de datos y fondos personales, presentar una auditoría anual por una empresa autorizada, así como el cumplimiento de los requisitos contra el lavado de dinero (Moorthy, 2018).

Es importante tener en cuenta que la regulación de las criptomonedas se está volviendo más estricta después del robo de 500 millones de dólares de la principal bolsa japonesa Coincheck en enero de 2018. Este se considera el robo más grande por parte de los piratas informáticos desde la llegada de las criptomonedas en 2009 (Arora, 2020).

Por tanto, las estrictas regulaciones impiden que las empresas de intercambio de bitcóin verifiquen sus credenciales de seguridad, lo que lleva a cierres de empresas. Como el mercado más grande de transacciones de bitcoines, Japón prefiere adoptar un enfoque estricto después del mayor robo. 


\section{Conclusiones}

La criptomoneda es una de las mayores innovaciones de FinTech y puede suponer una revolución sin precedentes a medida que vaya arraigando en la economía. Sin embargo, el alto grado de anonimato y la naturaleza descentralizada de la criptomoneda crean más problemas, como su uso en actividades ilegales, por ejemplo, la compra de armas, el narcotráfico, el financiamiento del terrorismo, etc. Su elevada rentabilidad y el fácil acceso al sistema también están impulsando su regulación. Desde el punto de vista jurídico-tributario, nacen diferentes aspectos problemáticos para abordar su calificación jurídica, la localización geográfica y el pseudoanonimato (Sedeño, 2020).

Los reguladores de todo el mundo han comenzado a trabajar en el marco legal del sistema. Algunos países han adoptado un enfoque de stand-by y esperar el desarrollo, mientras que un reducido grupo de países ha decidido adoptar un enfoque limitativo, imponiendo una prohibición general.

Por su parte, los legisladores deben proporcionar un marco legal muy claro y reglas para su implementación que lo complementen. Este marco legislativo debería comenzar con la integración de las criptomonedas en el régimen fiscal actual. Incluso si lo hace de manera indirecta, de acuerdo con la legislación vigente sobre impuestos a los productos básicos o sobre ganancias de capital, la implementación de reglas o lineamientos para su aplicación contribuirá a una mayor seguridad jurídica que es clave para el desarrollo económico.

Si las leyes existentes no clarifican el escenario de las monedas virtuales, los legisladores pueden elegir una legislación específica. Esto puede incluir enmiendas a las disposiciones legales existentes, pero la legislación y las enmiendas y adiciones deben ser lo más claras y concisas posible.

En un sentido más amplio, las reglas metodológicas también pueden incluir cómo se procesan otros criptoactivos con fines fiscales. Esto se debe a que las metodologías para criptoactivos o monedas son actualmente mínimas en muchos países, y desarrollarlas en paralelo puede ser realmente útil debido a su complejidad. No obstante, el vacío legal de los criptoactivos no significa una falta de regulación. Las actividades relacionadas con las criptomonedas están sujetas a tributación (Anglés, 2019).

También debemos considerar la necesidad de revisiones y adaptaciones frecuentes. Debido a la celeridad de la revolución tecnológica en la que nos vemos inmersos, cualquier metodología debe revisarse constantemente para seguir siendo influyente. También es necesario tener en cuenta los enfoques y tendencias internacionales de otros países. Al tomar cualquier decisión que no sea un debate natural, los reguladores deben explicar de manera clara e inequívoca las razones del régimen tributario adoptado o adaptado a la legislación existente. Una lógica de decisión clara puede hacerla más transparente y flexible, en el caso de que surjan nuevas monedas.

Además de desarrollar y actualizar la legislación, otro aspecto importante es monitorizar su implementación, cumplimiento y marco sancionador. La elevada volatilidad de las criptomonedas puede causar dificultades para su cumplimiento.

Otros problemas pueden surgir de diferentes tipos de cambio para la misma moneda virtual, la falta de evidencia de una conversión de moneda confiable en algunos casos y la necesidad de mantener registros de flujo de efectivo complejos y datos de transacciones. Las administraciones tributarias se enfrentarán a desafíos para obtener información confiable y oportuna sobre estas transacciones. En este sentido, un papel más fuerte en el seguimiento o alentar a los intermediarios a proporcionar información a las autoridades fiscales podría facilitar este proceso.

Otro aspecto que se debe tener en cuenta es cómo se armoniza el régimen tributario de las monedas digitales o cómo puede socavar los objetivos políticos adoptados a nivel nacional. Por ejemplo, las políticas gubernamentales para fomentar el uso de medios de pago electrónicos y reducir el uso de efectivo podrían conducir al desarrollo de monedas virtuales emitidas por los bancos centrales. Un verdadero reto para los órganos legislativos es adaptarse a la revolución Fintech y su velocidad. 


\section{Referencias bibliográficas}

ANA, M.; BUZZI, M.; MARIO, M.; CITTADINI, E. (2018). «Introducción a las criptomonedas». En: XXXIX Jornadas Nacionales de Profesores Universitarios de Matemática Financiera, págs. 1-23.

ANGLÉS, B. (2019). «La fiscalidad de Bitcoin en España». En: Crónica Tributaria, núm. 173, págs. 7-35 [en línea]. Disponible en: https://www.ief.es/vdocs/publicaciones/1/173.pdf\#page=7

ARORA, G. (2020). «Cryptocurrency Regulatory Framework in Japan». En: SSRN [en línea]. DOI: https://doi.org/10.2139/ssrn.3720230

BAL, A. M. (2014). Taxation of virtual currency. Doctoral thesis. Leyden University, Faculteit der Rechtsgeleerdheid [en línea]. Disponible en: https://scholarlypublications.universiteitleiden.nl/handle/1887/29963

BĂLĂȘOIU, N. (2021). «Perspectives on the Integration of Cryptocurrencies into National Tax Legislation». En: Scientia Moralitas International Journal of Multidisciplinary Research, vol. 6, núm. 1, págs. 40-47 [en línea]. Disponible en: http://scientiamoralitas.com/index.php/sm/article/view/86

BHATIA, A. (2021). "The discursive construction of legitimacy in the abrogation of Indian Constitution's Article 370». En: Journal of Pragmatics, núm. 183, págs. 132-141 [en línea]. DOI: https://doi. org/10.1016/j.pragma.2021.07.009

BOlotAEVA, O. S.; STEPANOVA, A. A.; ALEKSEEVA, S. S. (2019). «The Legal Nature of Cryptocurrency». En: IOP Conf. Series: Earth and Environmental Science, núm. 272 [en línea]. DOI: https://doi. org/10.1088/1755-1315/272/3/032166

BRAATEN, C. N.; VAUGHN, M. S. (2019). «Convenience theory of cryptocurrency crime: A content analysis of US federal court decisions». En: Deviant Behavior, vol. 42, núm. 8, págs. 958-978 [en línea]. DOI: https://doi.org/10.1080/01639625.2019.1706706

CVETKOVA, L. (2018). «Cryptocurrencies legal regulation». En: BRICS Law Journal, vol. 5, núm. 2, págs. 128-153 [en línea]. Disponible en: https://heinonline.org/HOL/LandingPage?handle=hein.journals/ brics5\&div=21\&id=\&page $=$

HUGHES, S. D. (2017). «Cryptocurrency Regulations and Enforcement in the U.S.». En: Western State Law Review, núm. 45, págs. 1-28 [en línea]. Disponible en https://heinonline.org/HOL/P?h=hein. journals/wsulr45\&i=7\%0Ahttps://heinonline.org/HOL/PrintRequest?handle=hein.journals/wsulr45\&collection=journals\&div=4\&id=7\&print=section\&sction=4

JIMÉNEZ, A. I. (2018). Criptomonedas y su fiscalidad. Universidad de La Laguna [en línea]. Disponible en: https://riull.ull.es/xmlui/bitstream/handle/915/9252/Criptomonedas\%20y\%20su\%20fiscalidad.pdf?sequence=1

KAZAKOV, A. (2018). Economic Analysis of Cryptocurrency. Universidad San Francisco de Quito USFQ [en línea]. Disponible en: https://repositorio.usfq.edu.ec/bitstream/23000/7674/1/140287.pdf

KONOWICZ, D. (2018). The new game: cryptocurrency challenges US economic sanctions. Technical Report [en línea]. Disponible en: https://apps.dtic.mil/sti/citations/AD1062142

LIANG, J.; LI, L.; CHEN, W.; ZENG, D. (2019). «Towards an understanding of cryptocurrency: A comparative analysis of cryptocurrency, foreign exchange and stock». En: IEEE International Conference on Intelligence and Security Informatics, págs. 137-139 [en línea]. DOI: https://doi.org/10.1109/ ISI.2019.8823373 
LISHCHYNSKA, V. O. (2019). «Legal Regulation of Cryptocurrency Circulation in The World». En: Comparative-Analytical Law, núm. 4, págs. 246-249 [en línea]. DOI: https://doi.org/10.32782/25240390/2019.4.68

MOORTHY, D. (2018). «A Study on Rising Effects of Cryptocurrency in the Regulations of Malaysian Legal System». En: International Journal of Business, Economics and Law, vol. 15, núm. 4, págs. 35-41 [en línea]. Disponible en: https://www.ijbel.com/wp-content/uploads/2018/05/LAW-71.pdf

NOVIKOV, A. (2018). «Legal Regulation of Cryptocurrencies and Applicable Risks». En: EUREKA: Social and Humanities, núm. 4, págs. 32-35 [en línea]. DOI: https://doi.org/10.21303/2504-5571.2018.00690

NOYA, E. (2016). «¿Es el fintech el mayor desafío que afronta la banca?». En: Harvard Deusto Business Review, núm. 254, págs. $22-29$ [en línea]. Disponible en: https://www.harvard-deusto.com/es-el-fintech-el-mayor-desafio-que-afronta-la-banca

SAMOLYSOV, P. (2020). «Legal Regulation of Cryptocurrency Mining». En: Law and Digital Economy, págs. 13-20 [en línea]. DOI: https://doi.org/10.17803/2618-8198-2020-09-3-013-020

SANZ-BAS, D.; DEL ROSAL, C.; NÁÑEZ ALONSO, S. L.; ECHARTE FERNÁNDEZ, M. Á. (2021). «Cryptocurrencies and Fraudulent Transactions: Risks, Practices, and Legislation for Their Prevention in Europe and Spain». En: Laws, vol. 10, núm. 3, 57 [en línea]. DOI: https://doi.org/10.3390/laws10030057

SEDEÑO, J. F. (2020). «El control tributario de las criptomonedas: calificación jurídica, localización geográfica y pseudoanonimato». En: Nueva fiscalidad, núm. 1, págs. 207-233.

SEREDA, I. M. (2020). «Features of the legal regulation of cryptocurrency: a foreign response». En: Вестник Сибирского Юридического Института МВД России, núm. 3, págs. 94-98 [en línea]. DOI: https://doi.org/10.51980/2542-1735_2020_3_94

SKOROKHOD, V.; LEONOVA, K.; NAHORNIAK, I. (2016). «Cryptocurrency in the Context of Development of Digital Single Market in European Union». En: InterEULawEast - Journal for International and European Law, Economics and Market Integrations, vol. 3, núm. 1, págs. 107-124.

VARGAS PORTILLO, P. (2020). «Influencers' recommendations on the Internet: effects of codes of conduct». En: Harvard Deusto Business Research, vol. 9, núm. 2, págs. 129-139 [en línea]. DOI: https:// doi.org/10.48132/hdbr.299

ZAERA VIDAL, G. (2014). Bitcoin: Bases, comportamiento como moneda e inversión. Universidade da Coruña [en línea]. Disponible en: http://hdl.handle.net/2183/13595 


\title{
Cita recomendada
}

GARCÍA-RAMOS LUCERO, Miguel Ángel; REJAS MUSLERA, Ricardo (2022). «Análisis del desarrollo normativo de las criptomonedas en las principales jurisdicciones: Europa, Estados Unidos y Japón». IDP. Revista de Internet, Derecho y Política, núm. 35. UOC [Fecha de consulta: dd/mm/aa] http://dx.doi.org/10.7238/idp.v0i35.391466

\begin{tabular}{|c|c|}
\hline SOMIERIGHIS RESERVED & $\begin{array}{l}\text { Los textos publicados en esta revista están -si no se indica lo contrario- bajo una licencia } \\
\text { Reconocimiento-Sin obras derivadas } 3.0 \text { España de Creative Commons. Puede copiarlos, } \\
\text { distribuirlos y comunicarlos públicamente siempre que cite su autor y la revista y la institu- } \\
\text { ción que los publica (IDP. Revista de Internet, Derecho y Política; UOC); no haga con ellos } \\
\text { obras derivadas. La licencia completa se puede consultar en: http://creativecommons.org/ } \\
\text { licenses/by-nd/3.0/es/deed.es. }\end{array}$ \\
\hline
\end{tabular}

\section{Sobre el autor \\ Miguel Ángel García-Ramos Lucero \\ EAE Business School \\ magarcia@campus.eae.es}

Doctorando en el programa de Ciencias Jurídicas y Económicas por la Universidad Camilo José Cela. Profesor de EAE Business School (Grupo Planeta). CFA Charterholder, máster en Finanzas por CUNEF y licenciado en Ciencias Económicas por la Universidad de Alcalá. Ha desempeñado relevantes cargos en distintas entidades financieras y ha publicado numerosos artículos en prestigiosas revistas académicas. Actualmente, centra su investigación en Fintech y criptomonedas.

ORCID: https://orcid.org/0000-0002-8671-0374

\author{
Ricardo Rejas Muslera \\ Universidad Camilo José Cela (Madrid) \\ rrejas@ucjc.edu
}

Licenciado en Derecho por la Universidad Carlos III de Madrid (1996), doctor por la Universidad de Alcalá (2007), acreditación positiva en las figuras de contratado doctor y profesor doctor de universidad privada (2011), Curso Superior de Abogados por la Escuela de Práctica Jurídica (1998), Curso de Experto en Derecho Societario por el ICAM (2000), colegiado ICAM (desde 2000) y director del Departamento de Derecho.

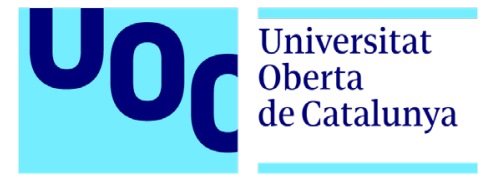

